\title{
aplicación de las resinas epoxi para revestimientos y juntas de hormigón, en los Estados Unidos
}

KURT WALZ, Düsseldorf. «Beton», 12, núm. 5, mayo 1962, pág. 215.

\section{Generalidades}

La California Division of Highways en Sacramento se ocupó ya en 1953, probablemente por primera vez en el mundo, de la utilización de resinas artificiales para trabajos de reparación en carreteras y puentes de hormigón. Debido a estas experiencias se han conocido las ventajas que ofrecen las resinas epoxi, y descubierto que se puede hacer también una buena soldadura entre hormigón viejo y nuevo mediante una capa de dichas resinas. También es de señalar que otras materias sintéticas, tales como el acetato de polivinilo, las resinas poliéster, las emulsiones de neopreno y las dispensiones en agua de copolímero estireno-butadieno, en juntas para hormigón, no se portan tan favorablemente como las resinas epoxi convenientemente preparadas. Estas últimas garantizan una soldadura fija y duradera entre partes de hormigón ya endurecidas y, también, entre hormigones recientemente preparados, como consecuencia de no ser sensibles a los álcalis. Sistemas de resina epoxi bien preparadas sueldan incluso sobre hormigón algo húmedo. Al endurecer retraen en una proporción insignificante, son resistentes a la humedad, y poseen una excelente resistencia contra las influencias atmosféricas, así como también contra todos los agentes químicos que normalmente entran a formar parte entre los componentes del hormigón. Con estas resinas se alcanza una mayor resistencia a la compresión y a la flexotracción que con el hormigón, y con un tratamiento especial adquieren, después de pocas horas, una rigidez suficiente para el uso. A causa del alto precio, de 2,5 a 5 dólares por litro, la resina epoxi, suministrada como líquido viscoso, se usa preferentemente en juntas como aglomerante y en reparaciones. Como aglomerante en masa se utiliza sólo en casos especiales para mayores reparaciones o complementos de partes de construcción fabricando un hormigón de resina epoxi con una relación de mezcla que se puede extender hasta 1:18 (resina epoxi-árido). En la reconstrucción del puente de San Francisco-Oakland Bay, actualmente aún en marcha, se han fabricado uniones relativamente compactas de hormigón de resina epoxi, porque dentro de pocas horas debe entrar en servicio.

En el precio de un metro cúbico de hormigón de resinas epoxi se hablaba de unos 260 dólares (Cal. Div. Highw.) hasta 540 dólares para una mezcla $1: 7$ (U. S. Bureau of Reclamation, Denver, USBR), según la relación de la mezcla. 
Según un informe de la Cal. Div. Highw. se emplea también una combinación más económica y de aplicación más extensa de Chemical Shell Co. (una resina epoxi líquida y una sustancia bituminosa, especialmente tratada-generalmente alquitrán de hulla-), para hacer revestimientos de poco espesor de superficies de carreteras y losas de calzadas de puentes estropeadas. Sin embargo, su color es oscuro. Según las experiencias, la adherencia sobre hormigón seco es muy buena, pero resulta relativamente mala sobre hormigón fresco; el endurecimiento tal vez sea muy lento, lo cual, a veces, es conveniente. Existen experiencias de varios años en el empleo práctico y buenos resultados de sistemas normales de resina epoxi realizados por la Cal. Div. Highw. Están realizándose o fueron realizados trabajos amplios por U. S. Bureau of Reclamation (informe más reciente: «Epoxy resins for concrete construction and repair. - Interim report Denver 1961»). El Departamento de las Fuerzas Aéreas (Dep. A. F.) editó instrucciones detalladas sobre la reparación de grietas y superficies de pistas de aeropuertos de hormigón, en unión con el Corps of Engineers, Dep. of the Army (Air Force Pamphlet, AFP 88-116-1; Washington 1959: Repairs to airfield rigid pavement).

\section{Composición}

El conglomerante de resinas epoxi se prepara, en el mismo lugar de empleo, a base del componente epoxi y una sustancia endurecedora (catalizador), ya que el proceso de endurecimiento empieza inmediatamente después de mezclarlos. Se dispone de un tiempo de elaboración de unos 20 a 45 minutos, según la sustancia endurecedora y la temperatura, y, por tanto, se debe preparar una cantidad limitada de conglomerante. La resina epoxi que se endurece sólo con catalizador se hace relativamente dura, y por su rigidez no siempre se la puede usar; por regla general, se añade además una sustancia de adición. Generalmente, se trata de polisulfuros (por ejemplo, tiocoles), que ya contienen la sustancia endurecedora, $o$, raras veces, de poliamidas, que también actúan como sustancias que endurecen al mismo tiempo.

En la Cal. Div. Highw., el conglomerante se compone, por ejemplo, de resinas epoxi en la siguiente proporción:

10 partes en peso de resina epoxi.

4 partes en peso de polisulfuros polimerizados (adición).

1 parte en peso de sustancia endurecedora.

Según las indicaciones del Departamento de las Fuerzas Aéreas, el conglomerante consta de dos partes de resina epoxi y una parte de polisulfuro incluida la materia que endurece. Se puede sacar como conclusión de los trabajos realizados en los tres centros antes mencionados, lo siguiente:

Como resinas epoxi se emplean las de los grupos diepoxi y poliepoxi.

Como sustancias que endurecen se usan el 2, 4, 6, tri (dimethylaminomethyl) fenol y el dimethylaminomethylfenol.

El USBR emplea como sustancia de adición, en primer lugar, polisulfuros o poliamidas. Los primeros contienen la sustancia que endurece (aminas primarias, secundarias o terciarias); las últimas no necesitan añadir la sustancia endurecedora, aunque en algunos productos comerciales están contenidas para refuerzo de ciertas propiedades.

Según los ensayos del USBR no se debe añadir un diluyente para rebajar la viscosidad y facilitar la elaboración, porque en todo caso se disminuyen las cualidades físicas del sistema endurecido (entre otras, menor densidad, fuerte contracción y pobres cualidades mecánicas).

La temperatura influye en gran medida en el tiempo necesario para el endurecimiento: temperaturas altas, acelerando, y bajas retardando. Ha dado muy buen resultado un aumento de la temperatura de la superficie del hormigón a la cual se aplica la resina epoxi y, a continuación, calentar la resina aplicada por medio de rayos infrarrojos. 
En la instrucción del Dep. A. F. se citan dos tipos de conglomerantes a base de resina epoxi, para temperaturas ambiente de 20 a $40^{\circ} \mathrm{C}$ y de 4,5 a $20^{\circ} \mathrm{C}$, que se distinguen probablemente por el catalizador añadido al polisulfuro.

Para las características de calidad del componente epoxi (entre otras, el peso específico, la viscosidad, el contenido en grupos epoxi) y de la sustancia de adición, incluso del material endurecedor (entre otros, contenido de agua, viscosidad, punto de inflamación, contenido de azufre), las instrucciones del Dep. A. F. citan valores límites y prescripciones detalladas de comprobación.

Repetidas veces se señala que se ofrecen en el mercado numerosos «ligantes de resinas epoxi», y que se pueden evitar fracasos sólo si la composición del sistema total está conforme con las peticiones y los conocimientos de empleo. Respecto a esto, son provechosas, sin duda, indicaciones tan detalladas como las publicadas por el Dep. A. F. y las preparadas por el USBR para sus programas de trabajo.

Los morteros de resinas epoxi se elaboran, según las indicaciones del Dep. A. F., con una parte de conglomerante de resina epoxi y 3 a 7 partes de arena, y los hormigones, de una parte de conglomerante y 6 a 10 partes de arena y grava.

\section{Propiedades}

Variados ensayos con morteros y hormigones de resinas epoxi permiten conocer, de acuerdo con los amplios programas del USBR, que con un proceder apropiado y un conglomerante adecuadamente compuesto se pueden lograr resistencias a la compresión de unos $1.200 \mathrm{~kg} / \mathrm{cm}^{2}$ y resistencias a la tracción de unos $90 \mathrm{~kg} / \mathrm{cm}^{2}$, casi independientemente de la relación de mezcla y el tamaño máximo del material inerte. Como resistencia inicial se habla, en las indicaciones del Dep. A. F. para conglomerantes de resinas epoxi, 20 horas después del endurecimiento a temperatura ambiente, de una resistencia a la compresión de $490 \mathrm{~kg} / \mathrm{cm}^{2}$ y una resistencia a la flexotracción de $70 \mathrm{~kg} / \mathrm{cm}^{2}$.

El módulo de elasticidad, según era de esperar, se influye en alto grado por la relación de mezcla, y se encuentra entre $140.000 \mathrm{~kg} / \mathrm{cm}^{2}$ y $335.000 \mathrm{~kg} / \mathrm{cm}^{2}$ (un buen hormigón de cemento oscila de 350.000 a $450.000 \mathrm{~kg} / \mathrm{cm}^{2}$ ). Es interesante señalar (después de algunos experimentos con mortero $1: 3$ y un conglomerante especialmente bueno), que e

miento hasta tensiones de unos $450 \mathrm{~kg} / \mathrm{cm}^{2}$; a parxistía proporcionalidad entre la tensión y el alargamente.

tir de aquí empezó la fluencia que crecía gradual-

Con muchos experimentos de flexión, compresión y cizalladura se confirmaba siempre la gran adherencia entre el conglomerante de resinas epoxi y el hormigćn viejo, como también entre el conglomerante de resinas y el mortero u hormigón recién preparados. Es norma que, con un proceder adecuado, la rotura no se produce a través de la superficie de la adherencia, sino a través de la masa de hormigón de cemento.

Según las experiencias del USBR se comprobaba una resistencia muy alta de estos conglomerantes a la formacićn de coqueras, y una resistencia al desgaste mecánico similar al hormigón de cemento o, a veces, superior.

Probetas cilíndricas, expuestas a 950 ciclos de hielo-deshielo, nos permiten conocer que es posible proteger el hormigón mal construido y, por tanto, delicado a las heladas, contra bruscas condiciones atmosféricas, mediante inyección de una película de resinas epoxi.

\section{Empleo}

Nos llevaría demasiado lejos, si pretendiésemos enumerar detalladamente todas las posibilidades de empleo y los distintos modos de trabajo. Aquí expondremos lo más esencial de las experiencias prácticas y de los ensayos, muy numerosos, del USBR. 
Las superficies de hormigón endurecidas, en las cuales se aplica conglomerante de resina epoxi (mortero u hormigón) directamente, deben estar limpias. En la práctica se ha demostrado como más efectiva la limpieza con chorro de arena. Igualmente, se alcanzaba buena adherencia si la superficie del hormigón, antes de la aplicación de una capa de resina epoxi, fue tratada con ácido clorhídrico diluido, lavada posteriormente y seca. Con superficies engrasadas o aceitadas es suficiente un tratamiento con un medio de limpieza adecuado $\mathrm{y}$, a continuación, chorro de arena o un lavado y, finalmente, un tratamiento con ácido. Las superficies deben estar secas en lo posible antes de la aplicación del conglomerante, mortero u hormigón; sin inconveniente, pueden estar. incluso, húmedas, pero nunca mojadas.

Si se usa una capa de conglomerante de resinas, sólo o en forma de mortero, como puente de adherencia entre hormigón viejo y nuevo, esta capa de adherencia, alisada o distribuida con un cepillo blando, debe adquirir muy poca rigidez antes que el mortero u hormigón fresco se apisone o vibre. Para lograr una buena unión, sin embargo, el hormigón o mortero fresco deben ser muy consistentes (asiento no mayor a $5 \mathrm{~cm}$ ).

Al aplicar morteros u hormigones de estas resinas en capas de más de $5 \mathrm{~cm}$ de espesor, como, por ejemplo, al igualar losas de calzada de diferente nivel, o al peraltar curvas de carreteras, se recomienda aplicar el mortero de resina epoxi en capas, a causa del desarrollo de calor durante el endurecimiento y poner la capa siguiente sćlo cuando la temperatura de la capa inferior ha bajado de nuevo considerablemente.

Una resistencia al deslizamiento suficiente en capas de mortero aplicadas como reparación sobre revestimientos de hormigón se alcanza, o bien haciendo muy fina la capa de mortero de la resina epoxi, o bien por fricción con arena o esmeril de la superficie fresca de la capa de resina epoxi. Sobre las losas de hormigón de puentes con grietas, sobre los cuales discurre el tránsito directamente, se han inyectado conglomerantes de resina epoxi y obtenido suficiente resistencia al deslizamiento esparciendo esmeril.

De este modo se protegía la construcción metálica cerrando las grietas, causa de una corrosión aumentada, por disolución de las sales de deshielo que penetraban en invierno.

Naturalmente, el conglomerante de resinas epoxi o sus morteros pueden crear una excelente unión muy adherente en anclajes o armaduras de conexión, o pueden pegar componentes prefabricados. Ejemplos de ello son: el alargamiento de pilotes hincados de hormigćn pretensado (Ben Gerwick, San Francisco) por medio de varillas pegadas por el centro y las superficies de junta revestida; y la unión de tramos de tubos centrifugados de $5 \mathrm{~m}$ de largo a pilotes tubulares pretensados de $50 \mathrm{~m}$ de longitud, para el puente de Cheasapeake Bay, cerca de Norfolk. Poniendo en rededor manguitos calentados eléctricamente se puede endurecer la junta de resina epoxi en pocas horas, y el pilote alargado se puede hincar más.

El cerrado de grietas con conglomerante de resina epoxi permite, bajo ciertas condiciones, unir de nuevo partes de la construcción agrietadas por las cargas. Repetidas veces se realizaron reparaciones de grietas, por ejemplo, en puentes de la Cal. Div. Highw. Como ejemplo especialmente interesante, de grandes reparaciones de grietas (unos $1.500 \mathrm{~m}$ en total), se cita la reconstrucción de una obra entera: los trabajos de reparación de una instalación de muelle de cuarenta años de edad, situada sobre pilotes hincados de hormigón, en el puerto de Los Angeles. Los apoyos principales, 0,75 $\times$ $\times 0,90 \times 16,5 \mathrm{~m}$, de la losa de hormigón armado sobre los cuales iban los ferrocarriles, mostraban grietas en las proximidades del apoyo encima de los pilotes, que eran verticales: en parte, finas, y en parte, muy fuertes. No se conocía la causa, aunque probablemente se trataba de un exceso de carga. Era necesaria una larga y penosa reconstrucción; después de inspeccionar las instalaciones, cuando se descubrieron las grietas, fue prohibido el tránsito; la inyección de resina epoxi líquida fue un remedio.

Por medio de sondeos se pudo comprobar que las superficies de las grietas eran limpias. Después de experimentos previos en diversas grietas y de comprobaciones en el laboratorio, se procedió de la manera acostumbrada: primero se fresaron las grietas (ranuras de 0,6/1,2 cm), y se hicieron agujeros a distancia de $25 \mathrm{~cm}$ (diámetro $19 \mathrm{~mm}$ ). En éstos se colocaron tochos de $19 \mathrm{~mm}$ de profundidad, con mortero de resina epoxi. Una vez endurecido el mortero de resina epoxi. que se había metido a 
la vez en la grieta, y que tapaba ésta hacia fuera, se inyectó toda ella con la resina líquida, empezando por la parte baja de los apoyos, con una presión hasta de unas 8,7 atm. Mientras tanto, se daba repetidas veces una presión de 6,3 atm. desde arriba sobre cada zona del relleno, por medio de un gas inerte, para rellenar todas las cavidades y grietas capilares. Tanto las características del conglomerante como las del mortero de cierre estaban fijadas hasta el último detalle (por ejemplo, composición y propiedades físicas de los componentes; resistencia a la compresión mín. $1.120 \mathrm{~kg} / \mathrm{cm}^{2}$; resistencia a la flexotracción mín. $700 \mathrm{~kg} / \mathrm{cm}^{2}$; resistencia a la tracción del conglomerante mín. $84 \mathrm{~kg} / \mathrm{cm}^{2}$ ).

Los testigos sacados después de la inyección de la obra confirman los hechos favorables, conocidos con experimentos anteriores. La resistencia a la tracción, transversal a la superficie de la grieta reparada, fue de $26,6 \mathrm{~kg} / \mathrm{cm}^{2}$, con pocas variaciones, algo mayor aún que en los experimentos previos, y mayor que en el hormigón inalterado $\left(19,5 \mathrm{~kg} / \mathrm{cm}^{2}\right)$. La rotura discurría en el hormigón fuera de la superficie pegada. Finalmente, se realizan mediciones en la obra con cargas estáticas y dinámicas. De los valores de estos resultados pudo deducirse igualmente la plena eficacia del cierre de las grietas.

Los gastos de la reconstrucción con conglomerante de resina y unos trabajos de inyección de hormigón fueron de 92.890 dólares; los de derribo con reconstrucción total de la instalación del muelle, incluyendo el déficit de los derechos de peaje, se habían calculado en 450.000 dólares.

\section{Conclusiones}

De esta breve descripción se puede sacar la conclusión que en los Estados Unidos existen investigaciones muy amplias y gran experiencia sobre el modo de proceder en el empleo de resinas epoxi para la reparación de hormigón, para la unión o el suplemento de obras de construcción, y para el relleno de juntas de transmisión de esfuerzos. Las investigaciones en Alemania, conocidas en algunos sitios, y los comienzos del empleo de resinas sintéticas como materias auxiliares de construcción deben aprovechar los conocimientos americanos. Debe advertirse que en el desarrollo americano se ha impuesto hasta ahora el conglomerante a base de resinas epoxi por sus grandes cualidades para el tipo de empleo que interesa a la ingeniería.

Para este empleo de resinas epoxi, que en Alemania aún está en sus comienzos, sería muy provechoso que la composición y cualidades técnicas de los diversos productos se dieran también a conocer al comprador, y pudieran juzgarse según un procedimiento de ensayo universal. Los experimentos del USBR hacen destacar sensiblemente que los «ligantes de resinas epoxi» pueden diferenciarse mucho en sus cualidades según el número de grupos epoxi y el peso molecular del componente resinoso, y según las otras sustancias empleadas para la composición del sistema (sustancias de adición, materiales endurecedores o diluyentes).

Las investigaciones de laboratorio y técnicas de empleo con un producto señalado sólo con una marca comercial, pero sin suficientes indicaciones sobre el sistema total del conglomerante o anunciado solamente como resina epoxi, no conduce a conocimientos de provecho general, ni tampoco a una selección apropiada en el futuro entre los productos ofrecidos, atendiendo a cualidades, preferibles en cada caso, de los diversos grupos de sistema.

\section{Bibliografía}

(1) Goldberger, H. W.: "Corrective measure employing epoxy resins on concrete bridge decks". Proc. Highw. Res. Board, 40, 1961, págs. 489-496.

(2) Wakeman, C. M.; STover, H. E., y E. N. BLYE: Glue for concrete repair, Materials Research \& Standards, 2, 1962, núm. 2, págs. 93-97. 